\title{
Condiciones de posibilidad para una justicia global
}

\author{
GUSTAVO PEREIRA \\ Universidad de la República, Uruguay
}

RESUMEN. El reconocimiento, en tanto que constitutivo de la subjetividad, es una necesidad básica tanto de las personas como de los Estados. Los términos de relación internacional impuestos por buena parte de los organismos internacionales de crédito y comercio niegan y socavan este reconocimiento a través de políticas que generan exclusión y pobreza en los países más pobres. La ruptura de esta lógica se presenta como un camino que posibilita el surgimiento de condiciones de justicia global basadas en términos de reconocimiento recíproco. Un argumento prudencial oficia como primer paso en este proceso, el beneficio a medio y largo plazo sería la motivación racional para que los organismos internacionales generasen una apertura que permitiese asegurar el reconocimiento recíproco. Sin embargo, este argumento no es suficiente y el objetivo de una inclusión radical que afecte a todas las regiones del planeta requiere un argumento moral basado en la idea de igual dignidad inherente a toda persona. La justicia global y su contraparte de justicia local solamente serán posibles a través de una conjunción de estos dos argumentos que habilitarían la instalación de una lógica de reconocimiento recíproco como posibilitante de la justicia.
ABSTRACT. Recognition, as a constitutive part of subjectivity, is a basic need for people as well as for States. The terms of international relationships imposed by a great number of international credit and commerce institutions contradict and undermine such recognition, by means of policies that result in segregation and poverty in the poorest countries. Breaking such logic seems to be the way to enable the existence of conditions of global justice based on terms of reciprocal recognition. A prudential argument is the first step in this process; mid and long-term benefit would be the only rational motivation for international institutions to enable such an openness that would ensure reciprocal recognition. However, this argument is not sufficient, and the objective of a radical inclusion affecting all regions of the planet requires a moral argument based on the idea of equal dignity, inherent to every person. Global justice and its counterpart, local justice, will only be possible through the combination of these two arguments, which would make the existence of a logic of reciprocal recognition possible, thus enabling justice. 


\section{Introducción}

H. G. Wells, en la Máquina del tiempo, profetizó al género humano dividido en dos especies: los Morlocks y los Eloi. Los primeros vivían bajo la superficie y como consecuencia de trabajar en la oscuridad habían quedado ciegos y se habían embrutecido. Por su parte, los Eloi eran una especie aristocrática que vivía en jardines alimentándose de fruta. Al estar ambos mundos comunicados, en las noches sin luna los Morlocks emergían a la superficie y devoraban a los Eloi.

Lo terrible de la metáfora de Wells está en que cada vez más se presenta como posible; no sería para nada excesivo sostener que nuestro mundo está dividiéndose en Morlocks y Eloi tanto a nivel local como global. La consecuencia más tangible de esta creciente división es la clausura del reconocimiento del otro. Los Morlocks no reconocen a los Eloi como un alter ego y por eso los devoran; son simplemente un medio para satisfacer una necesidad. Seguramente la metáfora nos está advirtiendo que la causa profunda de la creciente violencia local y global, de la inestabilidad, del riesgo, se sitúa en la negación de niveles mínimos de reconocimiento recíproco que deberían ser asegurados si es que nuestra existencia pretende seguir un rumbo diferente al profetizado por Wells.

En este trabajo mi intención es presentar, a la luz de una lógica de reconocimiento recíproco, algunas condiciones de posibilidad para una justicia global. Esta lógica de reconocimiento recíproco, que tiene en la ética del discurso a una de sus manifestaciones más distintivas, es negada por las políticas de los organismos internacionales que principalmente rigen la economía. La ruptura de esta dinámica que genera pobreza, marginación y exclusión, tanto local como global, requerirá de la introducción de argumentos que sean vinculantes para que los actores globales sienten las bases para el surgimiento de una justicia global. Para alcanzar tal meta no solamente se ofrecerá un argumento moral basado en la idea de igual dignidad inherente a todos los seres humanos, sino que también se apelará a un argumento prudencial que apunta a asegurar el propio beneficio a largo plazo.

\section{Reconocimiento recíproco como exigencia básica}

Dentro de la tradición hegeliana la necesidad de reconocimiento se presenta como una necesidad peculiarmente humana. Esto se debe a que la particularidad de un ser racional, autoconsciente, consiste en que no se complace exclusivamente con la satisfacción de sus apetitos biológicos, sino que debe ser reconocido por otros seres de su misma clase, confirmándole a través de su comportamiento el sentido de su propio valor. De ahí que todo ser ra- 
cional, en tanto que autoconsciente, es decir, consciente de sí mismo en sus acciones y por ende capaz de controlarlas, demanda reconocimiento.

En este sentido, la identidad solamente queda abierta a una constitución en términos de reconocimiento, en virtud de lo cual alguien se convierte en sujeto en tanto que es capaz de autocomprenderse a través de relaciones de reconocimiento recíproco con otros, y en particular con otros que tienen un rol significativo para él ${ }^{1}$. En la medida en que el reconocimiento es un rasgo constitutivo del sujeto, siempre será necesaria su presencia como elemento posibilitante de su condición misma de sujeto.

El hombre tiene un sentido de lo que se le debe como ser humano, como persona, y si no lo obtiene se siente rebajado ante sus propios ojos. Por lo tanto, el que otros hombres reconozcan que él también es un hombre, un ser racional y deliberante que, en consecuencia, no debe ser tratado como un mero instrumento, se convierte en una faceta tan básica como la satisfacción de los deseos materiales.

Si los hombres no fuesen seres autoconscientes necesitados de reconocimiento de sus semejantes, no habría relaciones propiamente sociales y morales entre ellos. Pero es posible que las reglas y las actitudes inherentes a estas relaciones sociales sean incompatibles con la satisfacción de esta necesidad, y es por esta razón que la necesidad de reconocimiento se convierte en regulatoria de las condiciones sociales que afectan a los sujetos. En la modernidad este hecho se manifiesta a través de políticas de reconocimiento igualitario que en las sociedades democráticas se encarnan en la positivación jurídica de derechos de igual participación política, de derechos sociales o de derechos relativos a diferencias de género o culturas.

Esta perspectiva, que coloca al reconocimiento como una necesidad básica inherente al proceso de constitución de la identidad del sujeto, habilita a que desde un enfoque kantiano se lo considere como algo que tiene "valor en sí» y que es propio de los «fines de la razón», y, por lo tanto, como un «fin que es deber». Esto nos conduce directamente a la problemática de la Metafísica de las costumbres, a su teleología y a la antroponomía que requiere.

\section{Eleuteronomía, antroponomía y procedimentalización}

Para comenzar, es necesario recordar que dentro de los parámetros de la ética kantiana todo ser racional, en virtud de esta condición, posee una voluntad autónoma, es decir, que es capaz de autolegișlar en concordancia con la ley moral. Este carácter autolegislativo de la voluntad racional es lo que la distin-

1 Cf. G. W. F. Hegel, La fenomenología del espíritu, México, FCE, 1987, pp. 113-115; Ch. Taylor, Argumentos filosóficos, Barcelona, Paidós, 1995, pp. 298-299; G. H. Mead, Mind, Self and Society, Chicago, University of Chicago Press, 1934, pp. 185-193. 
gue como voluntad libre, debido a que es capaz de someterse a sus propias leyes y, a su vez, determina su pertenencia al ideal regulativo del reino de los fines, «donde todos los seres racionales están sujetos a la ley de que cada uno de ellos debe tratarse a sí mismo y tratar a todos los demás, nunca como simples medios, sino siempre al mismo tiempo como fin en sí mismo» ${ }^{2}$. Por lo tanto, la autonomía, en tanto capacidad autolegisladora de seres racionales, será el fundamento de la dignidad humana y el suelo en el que se asienta la buena voluntad, ya que solamente en tanto que libre la voluntad será buena, puesto que solamente de esa forma es capaz de expresar su autonomía.

En función de esto, y en concordancia con la interpretación que ofrece Jesús Conill, la ética kantiana puede ser entendida como eleuteronómica, puesto que la moralidad es tal en tanto que expresión de la libertad, y solamente desde esta libertad tiene sentido la felicidad como componente del bien del hombre ${ }^{3}$. Pero en la medida en que como producto de la libertad del sujeto se encuentra la capacidad de proponerse fines, se vuelve necesario poder discriminar entre aquellos fines que son propios de la naturaleza del hombre y aquellos que surgen de una constricción de la voluntad. Solamente estos últimos califican como morales ya que son fruto de la capacidad autolegisladora del hombre, $y$, por lo tanto, el principio de la libertad, en la medida en que supone una constricción de la voluntad, debe ser entendido como autocoacción. En consecuencia, la libertad implica el dominio de las inclinaciones como forma de alcanzar los fines propios de la razón ${ }^{4}$.

Pero la posibilidad de discernir entre los fines que la libertad se propone no solamente demanda constricción en el sujeto, sino que también requiere una diferenciación inherente a los fines, ya que de no ser así todos los fines valdrían solamente como medios y en la medida en que no hubiese fines que valieran en sí, no sería posible la constricción a través del imperativo categórico. Según Kant, existen fines que tendrían esta característica de valer en sí, y, por lo tanto, serían objetos del libre arbitrio que debemos proponernos; éstos son fines que a la vez son deber ${ }^{5}$.

En tanto que la razón se defienda de la influencia de las inclinaciones sensibles que nos impulsa a ciertos fines, será necesario que proponga su propio fin, un fin moral, un fin de la razón pura, objetivamente necesario y en tanto tal, un fin que a la vez será un deber. De ahí que la voluntad racional para autodeterminarse libremente, además del momento formal que encarna en la universalidad, deba contar con una determinación por la materia moral que sería un fin racional y moral ${ }^{6}$.

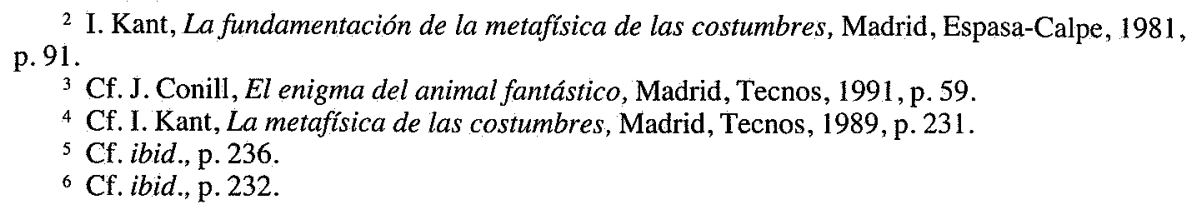

${ }^{2}$ I. Kant, La fundamentación de la metafisica de las costumbres, Madrid, Espasa-Calpe, 1981,

${ }^{3}$ Cf. J. Conill, El enigma del animal fantástico, Madrid, Tecnos, 1991, p. 59.

${ }^{4}$ Cf. I. Kant, La metafísica de las costumbres, Madrid, Tecnos, 1989, p. 231.

${ }^{5}$ Cf. ibid., p. 236.

${ }^{6}$ Cf. ibid., p. 232. 
La Tugendlehre señala un criterio para la legislación positiva: el fin que es a la vez deber establece una ley para las máximas de las acciones, porque exige subordinar los fines subjetivos a los objetivos; el fin que es deber nos obliga a convertir en deber la máxima correspondiente. Pero también queda un amplio espacio en manos del sujeto, porque es él quién debe decidir cómo y cuándo se debe obrar con vistas al fin que es a su vez deber, y será él también quien, en caso de conflicto entre máximas positivas, decida por cuál optar. El principio rector será entonces: «obra según una máxima de fines tales que proponérselos puede ser para cada uno una ley universal» 7 . Según la interpretación de Kant, el fin deja de ser meramente limitativo para ser propositivo; de la razón pura práctica parece deducirse un imperativo categórico que convierte a la humanidad en fin moral: es «un deber del hombre proponerse como fin al hombre en general», por lo que «el hombre es fin tanto para sí mismo como para los demás» ${ }^{8}$.

De la misma forma que la contradicción inherente al test del imperativo muestra sencillamente la imposibilidad, y los mandatos por ella acuñados son prohibiciones, los fines objetivos inspiran una legislación positiva, pero sin poder determinar a priori cuántas energías debe invertir el sujeto en perseguirlos ni cuáles debe elegir en el caso de que entren en conflicto. Esto amplía el margen de decisión de los individuos al interpretar el carácter de fin en sí mismo de cada hombre, no sólo como fin limitativo de las acciones humanas, sino como una tarea a realizar. En el humanismo eleuteronómico de la Metafisica de las costumbres, el hombre se propone como tarea y no sólo como límite restrictivo, lo que convierte a la ética kantiana en una verdadera antroponomía ${ }^{9}$.

Pero esta antroponomía adquiere una nueva dimensión bajo una perspectiva procedimentalista, de tal forma que si en la Metafísica de las costumbres el fin de la razón es el hombre en tanto sujeto racional, cuando la racionalidad ha pasado a ser una razón dialógica, los fines de la razón serán todos aquellos que aseguren la condición argumentante del sujeto.

A partir de la procedimentalización realizada por la ética del discurso, el proyecto kantiano puede ser entendido en términos dialógicos, y, en consecuencia, la libertad y la autonomía sólo pueden ser plenamente comprendidas una vez que son referidas a las condiciones de posibilidad de un diálogo. En esto interviene el proceso de descentración del sujeto autónomo y su transformación en un sujeto de reconocimiento recíproco, en virtud del cual solamente se puede dar cuenta de la autonomía en términos de identidad y reconocimiento. En tal sentido se vuelve necesario hablar de un nuevo suje-

\footnotetext{
${ }^{7}$ Ibid., p. 249.

8 J. Conill, El enigma del animal fantástico, op. cit., p. 64.

${ }^{9}$ Cf. A. Cortina, «Estudio preliminar a la metafísica de las costumbres», en I. Kant, La metafisica de las costumbres, op. cit., pp. LXXXII-LXXXIV; J. Conill, El enigma del animal fantástico, op. cit., pp. 65-67.
} 
to autónomo que asume las críticas al sujeto moderno como una potenciación del ideal emancipatorio ilustrado en lugar de una cancelación del proyecto.

\section{Sujeto autónomo reconstruido}

Las características del sujeto moderno como un sujeto con plena autodeterminación y plena autoconciencia que le vuelven autosuficiente, dominador de la naturaleza y autónomo han sido puestas en cuestión desde diversas perspectivas. Siguiendo a Wellmer, las críticas que convergen en esta noción de sujeto pueden agruparse según sus puntos de partida en:

1) La crítica psicológica del sujeto (desenmascaramiento) y de su razón; 2) la crítica filosófico-psicológico-sociológica de la razón «instrumental», o basada en una «lógica de la identidad», y de su sujeto; 3) la crítica de la razón transparente para sí misma, y de su sujeto constituyente de sentido, en la filosofía del lenguaje ${ }^{10}$.

La primera de estas críticas tiene como núcleo la negación empírica de la autonomía del sujeto y de su racionalidad, ya que el sujeto a partir del psicoanálisis es percibido como atravesado por fuerzas psíquicas y sociales que marginan su capacidad de autodeterminación a una condición de mediación entre tales fuerzas, la cual se realiza en el equilibrio siempre provisorio entre las demandas del Ello y las amenazas del Superyo.

La segunda perspectiva cuestionadora del sujeto moderno viene dada por la crítica a la razón instrumental que, si bien tiene a Nietzsche como antecedente significativo, tiene su manifestación más radical y estructurada en Adorno y Horkheimer. De acuerdo con la Dialéctica de la Ilustración, el sujeto es visto como la instancia opresora que es a la vez sometida, ya que el carácter sistematizador y unificador de la razón, que le permite instrumentalizar y dominar la naturaleza a la vez que autoconservarse, niega la propia naturaleza interna del sujeto dominada por impulsos no reducibles a la unificación de la razón ${ }^{11}$.

El tercer frente es el de la filosofía del lenguaje, donde lo que se cuestiona es el sujeto, quien a través de sus intenciones sería la fuente de las significaciones lingüísticas. La teoría referencial del significado implica que un signo lingüístico cobraría significación cuando un sujeto coordinara algo dado, la referencia, con un signo, por lo que la conexión que se supone que

\footnotetext{
${ }^{10}$ A. Wellmer, «Sobre la dialéctica entre modernidad y postmodernidad: crítica de la razón después de Adorno», en Sobre la dialéctica de modernidad y postmodernidad, Madrid, Visor, 1993, p. 74.

"T. Adorno y M. Horkheimer, Dialéctica del Iluminismo, Buenos Aires, Sudamericana, 1987,pp. 46-48.
} 
realizaría el sujeto se halla entre un nombre y una significación ya dada. Wittgenstein es quien descentra la significación del sujeto al remitirla a la noción de «juego de lenguaje». Este concepto debe ser entendido como una práctica intersubjetiva compartida determinada por un conjunto de reglas y que demanda que quien participa de ese juego sea adiestrado de tal manera que puede seguir esas reglas. La significación remite a estos juegos de lenguaje y sus reglas, por lo que, cuando se establece la significación como una relación de correspondencia entre el signo y el referente, lo que se está presuponiendo es una regla que se basa en la práctica de su aplicación a ciertos casos ${ }^{12}$. Por lo tanto, la significación no remite a elementos ya dados e independientes, sino a una práctica intersubjetiva que es la que fija la significación. En virtud de esto, la significación deja de centrarse en el sujeto y su intencionalidad, para asentarse en algo previo a toda instancia de este tipo, a saber, «juegos de lenguaje» o formas de vida, es decir, en un mundo que se abre lingüísticamente y que posibilita en tal apertura que los hombres puedan ser ellos mismos de diferentes maneras en cada caso ${ }^{13}$.

Este proceso de descentración del sujeto con sus tres posibles accesos conduce al descubrimiento de «lo otro» de la razón y del sujeto autotransparente en el interior mismo del sujeto, en lo irreductible a la razón instrumental y en la praxis intersubjetivamente constituida previa a toda significación. Esta praxis comunicativa, que es constitutiva del sentido, es el nuevo suelo desde donde deberá repensarse los conceptos distintivos de la modernidad. Es a partir de un supuesto de sujeto sometido a la crítica de la modernidad vuelta sobre sí misma que surgirá una nueva idea de autonomía irreductible a la unicidad y abierta a la diferencia, un sujeto que a través de un uso comunicativo del lenguaje manifiesta su carácter irreductible a las prácticas institucionales cosificadoras que lo someten a la unicidad del concepto, en tanto que el sujeto es entendido como mero objeto y no como un co-sujeto.

En particular, las políticas públicas locales y globales parten de supuestos negadores de la diversidad y realizan, a través del imperio de «lo idéntico», una fuerte uniformización de necesidades cosificando a los afectados. Es decir, en tanto que los afectados son sometidos a una relación sujeto-objeto, se da esa relación de uniformización propia de una racionalidad que tiene a los modelos fisicalistas como paradigma. Solamente una apertura comunicativa de tal racionalidad permitirá ingresar a los afectados a una dimensión sujeto-sujeto, introduciendo su irreductible diferencialidad y posibilitando un tratamiento del otro en concordancia con la variabilidad intersubjetiva.

${ }_{12}$ Cf. L. Wittgenstein, Investigaciones filosóficas, México, Unam-Crítica, 1988, pp. $23-29$.

${ }^{13}$ Cf. A. Wellmer, «Sobre la dialéctica entre modernidad y postmodernidad», op. cit., pp. 84-85. 


\section{5. Ética de la responsabilidad solidaria}

En este punto es necesario volver a la procedimentalización de la perspectiva kantiana realizada a partir de la ética del discurso, y en tal sentido es imprescindible recordar que bajo estos parámetros la libertad y la autonomía pasan a ser inteligibles en tanto que referidas a las condiciones de posibilidad del diálogo. Estas condiciones de posibilidad requieren la introducción de lo que Apel ha denominado la parte B de la fundamentación de la ética del discurso. Pero previo a ello es necesario recordar que en la propuesta de Apel los presupuestos pragmático-trascendentales de la argumentación, que son irrebasables para todo el que participe en un diálogo, posibilitan en primer lugar la fundamentación de un principio de universalización (U), que adopta el papel de una regla de argumentación y por el cual

toda norma válida ha de satisfacer la condición de que las consecuencias y efectos laterales, que del seguimiento general de la norma previsiblemente se sigan para la satisfacción de los intereses de cada uno, puedan ser aceptados sin coacción por todos los afectados ${ }^{14}$.

Y también tales presupuestos pragmático-trascendentales permiten fundamentar un principio de corresponsabilidad transcendental que afecta a cada potencial argumentante, en tanto que copartícipe del discurso, y que lo compromete a cooperar en la identificación y solución de los problemas moralmente relevantes que pueden y deben tematizarse en discursos prácticos con arreglo al principio $U^{15}$. Esta corresponsabilidad, en tanto que inherente a los presupuestos pragmático-trascendentales de la argumentación, precede transcendentalmente a todas las instituciones sociales, y en función de esto es que Apel sostiene que puede identificarse con la «responsabilidad humana para la creación histórica y cambios necesarios de las instituciones sociales» ${ }^{16}$. Esto conlleva la corresponsabilidad de dar solución a aquellas situaciones donde los discursos prácticos con arreglo al principio $U$ no pueden realizarse debido a que los participantes de la interacción, como consecuencia de las circunstancias que los afectan, no pueden intervenir en los discursos. En función de esto es por lo que la ética del discurso debe complementar su parte A de corte ideal, caracterizada por el compromiso procedimental con el principio U, con una parte B comprometida con la utilización de la racionalidad estratégica orientada a modificar las condiciones que

\footnotetext{
14 J. Habermas, Escritos sobre moralidad y eticidad, Barcelona, Paidós/ICE/UAB, 1987, p. 68.

15 Cf. K.-O. Apel, «How to Ground a Universalistic Ethics of Co-responsibility for the Efeects of Collective Actions and Activities», Philosophica, 52, 2, 1993, pp. 9-29.

16 K.-O. Apel, «Globalización y necesidad de una ética universal», en A. Cortina y D. GarcíaMarzá, Razón pública y éticas aplicadas, Madrid, Tecnos, 2003, pp. 195-196.
} 
obstaculizan la posibilidad de llegar a soluciones discursivas de los problemas morales. En esta parte B de la ética del discurso, el principio U se convierte en un valor que puede oficiar como parámetro para un principio teleológico de complementación ${ }^{17}$.

Por lo tanto, la parte B de la ética del discurso al proponer un principio teleológico se convierte en la guía para esa procedimentalización de la antroponomía inherente a la ética kantiana que se ha manejado. De ahí que el telos de la comunicación, entendido como la realización de los discursos prácticos en conformidad con el principio $U$, es el parámetro que permitirá asegurar las condiciones de realización de la autonomía y la libertad. En tal sentido, la necesidad de reconocimiento como sujeto capaz de argumentación se vuelve regulativa, realizando este criterio un corte al interior de las distintas demandas de reconocimiento y calificando solamente a aquellas que hacen a la condición misma de sujeto.

De esta forma, la ética del discurso permite reeditar la antroponomía inherente al proyecto kantiano bajo el supuesto de un sujeto moderno reconstruido que posibilitará escapar al reduccionismo del pensamiento identificatorio y a la lógica de la racionalidad instrumental. A continuación se presentará un criterio que permita transitar desde la antroponomía a la evaluación de políticas de justicia específicas.

\section{Autonomía como guía contrafáctica}

En tanto que solamente en términos dialógicos podemos hablar de un sujeto autónomo constituido bajo los parámetros del reconocimiento recíproco, la procedimentalización de la antroponomía requiere asegurar todo aquello que opere como condición posibilitante del diálogo. En consecuencia, el telos de la comunicación se convierte en la guía que permitirá asegurar las condiciones de realización de la autonomía y la libertad, por lo tanto, como ya se ha afirmado, la necesidad de reconocimiento como sujeto capaz de argumentación se vuelve regulativa, puesto que asegurar las condiciones necesarias para el reconocimiento implica asegurar las condiciones de posibilidad del diálogo.

Con respecto a estas condiciones de posibilidad estamos obligados a promover su realización y para ello es necesario una guía que especifique la autonomía y la libertad. En tal sentido, el enfoque de las capacidades de Amartya Sen brinda tal guía por tres razones:

${ }^{17}$ K.-O. Apel, «La ética del discurso como ética de la responsabilidad. Una transformación posmetafísica de la ética de Kant», en K.-O. Apel, Teoría de la verdad y ética del discurso, Barcelona, Paidós/ICE/UAB, 1991, p. 181; Diskurs and Verantwortung, Frankfurt a. M., Suhrkamp, 1988, pp. 146-150. 
a) En primer lugar, porque parte del mismo supuesto de sujeto, es decir, un sujeto no solamente capaz de determinar su plan de vida en clave de optimización de la utilidad personal, sino también capaz del reconocimiento de las bases de la cooperación social que se especifica a través de la dimensión del compromiso que tiene todo sujeto ${ }^{18}$.

b) Pero también porque supone a la libertad y la autonomía como lo propio del hombre, lo que, al decir de Conill, la vuelve una propuesta eleuteronómica que constituye una de las versiones neokantianas contemporáneas más destacadas junto con la ética del discurso y el constructivismo de Rawls ${ }^{19}$.

c) Por último, el tercer rasgo que permite vincular el enfoque de las capacidades a la ética del discurso se encuentra en la sensibilidad a la diferencia que comparten ambas propuestas y que sienta las bases para la construcción de sujetos de reconocimiento recíproco. El enfoque de las capacidades al establecer el tratamiento igualitario respetando la radical diferencialidad intersubjetiva, y por ende al asegurar el debido reconocimiento de todos los afectados, genera las condiciones para la constitución de la subjetividad en términos de reconocimiento recíproco.

En resumen, el enfoque de las capacidades se presenta como una propuesta que posibilita la implementación de una guía para la toma de decisiones, evaluación y diseño de políticas, en términos de la autonomía del sujeto entendida en clave de reconocimiento recíproco.

El concepto de capacidad es de importancia central para el diseño de esta guía, y para realizar su presentación conceptual optaré por hacerlo en referencia al concepto de funcionamientos. Para Sen, la vida puede concebirse como un conjunto de funcionamientos, es decir, un conjunto de estados y acciones, donde la realización de una persona puede entenderse como el vector de sus funcionamientos, abarcando dichos funcionamientos cosas tales como por ejemplo, «estar bien alimentado», «tener buena salud», «tener dignidad», «participar en la vida de la comunidad», etc. Los funcionamientos son constitutivos del estado de una persona, y la evaluación del bienestar de una persona depende de cómo estos elementos sean evaluados. Por su parte, la idea de capacidad representa las diversas combinaciones de funcionamientos que puede realizar una persona; por lo tanto, la capacidad

18 A. Sen, «Rational Fools: A Critique of the Behavioural Foundations of Economic Theory», Philosophy and Public Affairs, 6, 4, 1977. Reeditado en A. Sen, Nueva economia del bienestar. Escritos seleccionados, Valencia, Universitat de València, 1995, pp. 83-102. He presentado este argumento para justificar un criterio basado en la autonomía que permita romper con la lógica de las preferencias adaptativas. Cf. G. Pereira, «Autonomia, preferencias adaptativas y justicia distributiva», Sistema, núm. 178, enero de 2004, pp. 71-86.

19 Jesús Conill coincide con esta interpretación al presentar el concepto de libertad en Sen estrechamente ligado a la eleuteronomía en Kant. Cf. J. Conill, «Bases éticas del enfoque de las capacidades de Amartya Sen», Sistema, núm. 171, noviembre de 2002, pp. 47-63. 
de una persona refleja su libertad para llevar adelante un tipo de vida $u$ otro ${ }^{20}$. En función de esto, las capacidades pueden actualizarse a través de un haz de funcionamientos, quedando a discreción del sujeto la posibilidad de tal actualización; la libertad de una persona, en tal sentido, se encuentra a nivel de esa potencialidad que otorgan las capacidades de actualizar diferentes funcionamientos.

La construcción de una guía que posibilitara la evaluación y el diseño de políticas públicas locales y globales requiere la identificación de un conjunto de capacidades que permitirá a través de su ejercicio alcanzar los funcionamientos que posibiliten asegurar la condición de autonomía en términos de reconocimiento recíproco, así como también expandir la libertad efectiva de los afectados y evitar el menoscabo del reconocimiento a través de la reducción de la diversidad a criterios unificadores.

Como conjunto tentativo de capacidades básicas tomaré las que Martha Nussbaum ha presentado. Ellas son:

1. Ser capaz de vivir una vida completa, no morir prematuramente.

2. Ser capaz de tener buena salud, de estar bien alimentado, de tener adecuada vivienda, de tener oportunidades de satisfacción sexual, de moverse hacia diferentes lugares.

3. Ser capaz de evitar el dolor innecesario y de tener experiencias placenteras.

4. Ser capaz de usar los cinco sentidos; ser capaz de imaginar, de pensar y de usar la razón.

5. Ser capaz de experimentar apego a cosas y personas fuera de nosotros mismos; en general, amar, llorar la muerte de alguien, extrañar y sentir gratitud.

6. Ser capaz de formar una concepción del bien y reflexionar críticamente acerca de la planificación de nuestra vida.

7. Ser capaz de vivir por y con otros, de reconocer y mostrar consideración por otros seres humanos, de tomar parte en interacciones sociales y familiares.

8. Ser capaz de sentir consideración por y en relación con animales, plantas y la naturaleza.

9. Ser capaz de humor, de juego y de disfrutar actividades recreativas.

10. Ser capaz de vivir la propia vida, y no la de alguien más, en el contexto que le ha tocado ${ }^{21}$.

${ }^{20}$ Cf. A. Sen, Nuevo examen de la desigualdad, Madrid, Alianza, 1995, pp. 53-54

${ }^{21}$ Cf. M. Nussbaum, «Human Functioning and Social Justice», Political Theory, 20, 2, 1992, pp. 220-224; Women and Human Development, Nueva York, Cambridge University Press, 2000 , pp. 74-82. David Crocker también presenta un conjunto tentativo que coincidiría a grandes rasgos con el de Nussbaum. Cf. D. Crocker, «Consumption, Well-Being and Capability», en D. Crocker y T. Linden (eds.), Ethics of Consumption. The Good Life, Justice and Global Stewardship, Nueva York, Rowman \& Littlefield Publishers, 1998, pp. 336-390. 
Nussbaum también introduce la idea de un umbral interno a cada capacidad que delimitaría el mínimo nivel de desarrollo de capacidades necesario para adquirir la condición de autonomía del sujeto. Esto habilita a la introducción de la distinción conceptual entre autonomía potencial y plena fundada en diferencias de desarrollo en el conjunto de capacidades básicas.

Esta idea de potencialidad, determinada por un bajo nivel en el desarrollo de capacidades, significa que si los afectados fuesen sometidos a políticas compensatorias que apuntaran al desarrollo de capacidades, necesariamente alcanzarían el estado de autonomía plena. Esta relación entre potencialidad y plenitud genera el deber de que las políticas públicas apunten a superar esa brecha. La razón para esto último se asienta en que un desarrollo mínimo de capacidades básicas se presenta como condición de posibilidad de toda situación de diálogo, y, a su vez, en el ejercicio de su condición de argumentante se encuentra lo propio de un sujeto autónomo constituido en términos de reconocimiento recíproco.

Es necesario indicar que la lista es una lista abierta y general, que permite variaciones en su aplicabilidad, tanto por las múltiples especificaciones de cada uno de sus componentes, como por las distintas sociedades a las que se aplique. Esta variabilidad no afecta al conjunto de capacidades básicas, sino que lo especifica, por lo tanto, la variabilidad del umbral dependerá de lo que cada sociedad considere como mínimos de dignidad, y es justamente esta variabilidad la que permite asegurar que las bases del reconocimiento sean aseguradas transculturalmente. Asimismo, el mínimo de capacidades básicas podrá ser expandido en la forma que cada sociedad considere pertinente, por lo que la expansión de la libertad se orientaría en función de la diversidad de cada sociedad y de sus características propias en lo que hace visiones comprehensivas, pero siempre asegurando universalmente el mínimo que permite contar con sujetos autónomos.

De esta forma, el conjunto de capacidades básicas que son condición de posibilidad de un sujeto autónomo, en tanto que sujeto argumentante de reconocimiento recíproco, nos permite contar con una guía que posibilita tanto la evaluación de las políticas de justicia global, como el diseño de una alternativa.

\section{Organismos internacionales y la reducción de la diversidad}

En este punto quiero considerar la lógica que subyace a las políticas de los organismos internacionales de más destacados; si bien más adelante me referiré a los de comercio, por el momento consideraré exclusivamente a los de crédito.

Para comenzar, sostendré que es de suponer que los organismos internacionales de crédito tienen como una de sus metas propiciar el desarrollo 
económico de los países a quienes otorgan crédito, y que a ello apuntan también sus exigencias sobre la economía local que son la contraparte de sus préstamos y que hacen, por ejemplo, al déficit fiscal, la inflación y la política cambiaria, entre otros. Quiero referirme a este tipo de medidas como medidas reductivas de la diversidad, y para fundamentar esto último apelaré a las herramientas conceptuales que Adorno y Horkheimer nos han legado a partir de su diagnóstico de la Modernidad en la Dialéctica de la Ilustración. Creo que sus posiciones, aunque no seguidas hasta sus últimas consecuencias, son de suma utilidad para explicar la dinámica de reconocimiento y negación del mismo, inherente a las políticas de tales organismos.

Una de las tesis centrales que Adorno y Horkheimer presentan sostiene la confluencia de la racionalidad formal y la racionalidad instrumental. Esto significa que la racionalidad formal conduce a la sistematización del saber que se instala en todas las realizaciones cognoscitvas y modos de operar de los hombres, siendo a su vez esta racionalidad una racionalidad instrumental que, en tanto que cosificadora, apunta al control y la manipulación de procesos sociales y naturales. Tanto los seres humanos como la naturaleza se convierten en objetos de descripciones y explicaciones para una lógica que se rige por la cuantificación y la causalidad, y que tiene como consecuencia la equiparación de los sujetos a objetos inmateriales ${ }^{22}$.

Por lo tanto, en la medida en que la naturaleza y su conocimiento se convierten en paradigmáticos, la realidad social de los seres humanos será captada de acuerdo a la lógica de esta forma de conocimiento, equiparándose la naturaleza inorgánica a la naturaleza humana, y convirtiéndose la sociedad en un sistema de funciones y los hombres en cosas manipulables. A través de este proceso se asentaría la fuerza identificatoria de la razón instrumental, que cosifica a los hombres reduciendo la diversidad a lo uno y clausurando toda diversidad.

Como más arriba se ha presentado, esta crítica contribuyó en forma determinante al proceso de descentración del sujeto, que supone la aplicación de la crítica moderna a la propia modernidad, y que posibilita una alternativa a la racionalidad instrumental en términos de múltiples racionalidades que tienen la particularidad de dar cuenta de lo diverso, de aquello que es irreductible a lo uno.

La alternativa de una racionalidad comunicativa que rompe con la relación privilegiada sujeto-objeto propia de la racionalidad instrumental para centrarla en la relación sujeto-sujeto a través de una praxis comunicativa intersubjetivamente vinculante es lo que posibilitará la constitución de un punto de vista desde donde evaluar las políticas institucionales cosificadoras que tienen por particularidad la reducción de la diversidad.

${ }^{22}$ Cf. T. Adorno y M. Horkheimer, Dialéctica del Iluminismo, op. cit., pp. 19-20. 
En consonancia con la lógica de la racionalidad instrumental, las políticas de los organismos internacionales de crédito parten de supuestos negadores de la diversidad y aplican criterios de uniformización a través de sus exigencias a las economías locales. La reducción de la diversidad en la aplicación de estas políticas tiene consecuencias tales como la anulación del peso de la historia económica de cada país, de la particularidad de modalidades de producción, del peso de la tradición cívica, del valor que puedan tener las empresas del Estado para una comunidad, así como también la imposición de metas a alcanzar en inflación, déficit fiscal, etc. Tanto el FMI como el Banco Mundial cosifican, a través de sus políticas, a las metas macroeconómicas que imponen, sin prestar mayor atención a aquello que se supone que tales políticas pretenden proteger o asegurar. Es decir, el desarrollo, el bienestar, el empleo se convierten en medios para asegurar el fin de lograr metas macroeconómicas que supuestamente indicarían que una economía está bien encaminada. Una verdadera transvaloración se instala a nivel de la lógica de la racionalidad instrumental transformando fines en medios y viceversa, y logrando que lo que tiene valor en sí y que no admite valor de cambio alguno, es decir, que tiene dignidad, pase a ocupar el lugar de aquello que tiene precio y, por lo tanto, es negociable ${ }^{23}$. En tal sentido, la cuantificación inherente a este proceso de reducción de la diversidad a lo uno, y de transvaloración de lo que tiene dignidad por lo que tiene precio, conduce a que la pobreza, el hambre, la cobertura sanitaria, la inversión en educación sean espacios sacrificables en aras de lograr estas metas macroeconómicas impuestas por los organismos internacionales. Detrás de estas categorías no hay personas, no hay nada que tenga dignidad, no hay nada que tenga valor en sí, sino que simplemente son cifras a ajustar para que la economía «funcione bien» y «a largo plazo beneficie a todos». El categórico fracaso de estos proyectos, que sin duda tiene como ejemplo paradigmático el haber colocado a Argentina, el quinto productor de alimentos del mundo, en una situación de pobreza alarmante, nos obliga a replantear esta dinámica de relacionamiento y a buscar una alternativa.

Esta lógica cosificadora inherente a la racionalidad instrumental puede ser subvertida, es decir, puede ser re-transvalorada a partir de una lógica que rompa con el primado de la relación sujeto-objeto introduciendo la relación sujeto-sujeto. Esta lógica que supone a un otro no cosificado, a un otro reconocido recíprocamente, traerá consigo la diversidad inherente a cada particularidad, básicamente porque nadie mejor que el propio afectado para dejar de manifiesto sus propios intereses.

En la medida en que los países afectados en tanto que agentes ${ }^{24}$ son cosificados a través del relacionamiento que establecen los organismos

${ }^{23}$ Para ver este proceso de transvaloración como inherente a la lógica de la racionalidad instrumental. Cf. J. Conill, El enigma del animal fantástico, op. cit., pp. 13-17.

${ }^{24}$ En lo que hace al estatus de agente que le corresponde a los Estados, Dworkin realiza un excelente desarrollo que toma fuerte distancia del individualismo metodológico a través de la per- 
internacionales de crédito, se da una relación de uniformización propia de una racionalidad que tiene a los modelos fisicalistas como paradigma. Solamente una apertura comunicativa de tal racionalidad posibilitará que los Estados afectados ingresen en una relación sujeto-sujeto que habilite la introducción de su irreductible diferencialidad y que siente las bases para una justicia global.

En función de lo anterior, la búsqueda de condiciones posibilitantes de una justicia global deberá contar con dos momentos. El primero de ellos es el que hace a la simetría en las relaciones entre los organismos internacionales y los países afectados. Esto no solamente requiere que los Estados sean escuchados, sino que también es necesario que los organismos internacionales tengan una apertura al diálogo que hasta ahora no tienen. Esta apertura, en la medida que introduce la simetría en la relación, significa entre otras cosas asumir la posibilidad de la falibilidad en el diseño de políticas, lo que habilitaría a un proceso de ruptura de dogmas que tendría como principal consecuencia la inclusión de otros enfoques aparte del dominante como posibles alternativas a considerar en el diseño de sus políticas. Por lo tanto, la inclusión de la diferencia demandará el reconocimiento del otro como un co-sujeto, como alguien cuyos puntos de vista pueden y deben ser tenidos en cuenta en el diseño de las políticas. A su vez, este reconocimiento, en tanto el otro es considerado como un co-sujeto y sus argumentos pesan en términos de igualdad, contribuirá a la inclusión de otros enfoques en el diseño de las políticas con el objetivo de asegurar las bases del reconocimiento recíproco. Esto significaría que no solamente serían escuchados, sino que serían verdaderos interlocutores en la medida en que sus posiciones podrían ser aceptadas y modificar el dogma.

Un segundo momento de las condiciones posibilitantes de la justicia global hace a lo que se encuentra detrás de cada Estado, porque detrás de este agente reconocido internacionalmente se puede esconder la mayor injusticia local. En función de esto, un diseño de políticas por parte de los organismos internacionales en términos de justicia y bajo el supuesto de un igual reconocimiento a los países afectados, si no atiende a la justicia local puede tener como consecuencia la profundización de las diferencias sociales y la reproducción de la injusticia. Pero, sin embargo, y a partir de esto último,

sonificación del Estado y de la integración de la sociedad en torno a principios constitutivos. Éste es un supuesto metodológico que posibilita que dicha comunidad o Estado sea considerado como un tipo especial de entidad diferente de las personas verdaderas que son sus ciudadanos, atribuyéndole acción moral y responsabilidạd. Una de las virtudes más destacables del tratamiento de Dworkin es que evita todo riesgo de primado ontológico de la comunidad asentando la integración en hechos propios de las prácticas sociales que realizan los individuos. Los miembros reconocen una unidad de agencia personificada en la que no son individuos sino componentes, siendo esa vida colectiva de la comunidad la compuesta por aquellas actividades que consideran constitutivas de su vida colectiva. Cf. R. Dworkin, El imperio de la justicia, Barcelona, Gedisa, 1992, pp. 126 y ss.; La comunidad liberal, Bogotá, Siglo del Hombre Editores, 1996, pp. 161-174. 
los organismos internacionales en función del rol que cumplen tienen una formidable herramienta para la construcción de la justicia local, que no es más que un estadio de la justicia global. Si se suele exigir como contraparte del crédito otorgado objetivos a nivel de la macroeconomía, por qué no exigir también logros en lo que hace a justicia social, por ejemplo, sanidad, remuneración en ciertas áreas, empleo, niveles de educación, etc. Como criterio para estos mínimos exigibles contamos con un conjunto de capacidades básicas que tienen por rasgo distintivo el ser posibilitantes de la condición de autonomía plena entendida en términos de reconocimiento recíproco, $y$, por lo tanto, son también las condiciones básicas para el ejercicio de la ciudadanía en las sociedades democráticas.

Pero estos dos momentos de las condiciones de la justicia global son fuertemente interdependientes, porque el desarrollo de capacidades básicas contará como un objetivo global solamente si la lógica cosificadora que transvalora aquello que tiene dignidad en un simple medio es modificada. De ahí que el reconocimiento recíproco como base para el relacionamiento internacional se presente como un objetivo global con fuerte impacto local.

Una pregunta surge en este momento, y es por qué razón los organismos internacionales habrían de propiciar el surgimiento de estas condiciones de justicia global. De la respuesta dependen buena parte de las perspectivas de éxito de una empresa de este tipo.

\section{Razones para la justicia global}

La pregunta de por qué habrían los organismos internacionales de actuar de la forma indicada más arriba, asegurando el reconocimiento recíproco e incluyendo a la justicia como una condición a cumplir por parte de quienes reciben sus beneficios, tiene una respuesta con dos argumentos: uno moral y otro prudencial.

El argumento moral se asienta en la idea de igual dignidad propia de la autocomprensión moderna del hombre y que, en palabras de Kant, coloca al ser humano como un fin en sí mismo que no puede intercambiarse por nada, y por esto el ser humano tiene dignidad y no precio. En virtud de esta igual dignidad propia de sujetos autónomos y libres es que debe asegurarse todo aquello que la posibilite. Un conjunto de capacidades básicas, como hemos indicado, se convierte en una excelente guía para diseñar políticas que apunten a asegurar la autonomía y la libertad como componentes esenciales de estos sujetos de reconocimiento recíproco.

Pero las posibilidades de asegurar el desarrollo de capacidades se encuentran fuertemente condicionadas por los términos de relación internacional que afectan a los Estados; esto es, políticas de crédito, políticas comerciales, etc. Por lo tanto, la búsqueda de un trato igualitario entre los 
diferentes países con los organismos internacionales tiene el objetivo de reducir el impacto que este condicionamiento internacional tiene en la constitución de sujetos autónomos a nivel de cada sociedad en particular. Dentro del sistema de relaciones internacionales las políticas que socavan en mayor medida las posibilidades de que los gobiernos locales propicien un desarrollo de capacidades básicas son las que tienen que ver con el crédito y el comercio, por lo tanto, organismos como la OMC, el Banco Mundial y el FMI tienen un rol protagónico a cumplir si es que esta idea de igual dignidad resulta vinculante.

A su vez y como ya se ha señalado, si el objetivo de asegurar ciudadanos plenamente autónomos que doten de sustento a las democracias es tomado por estos organismos, en función del rol que éstos cumplen tienen una poderosa herramienta para exigir a nivel local el desarrollo de capacidades básicas como forma de reducir la injusticia. Podrían convertirse en verdaderos promotores del desarrollo y la justicia.

Pero a fuerza de ser sincero, no creo que este tipo de argumentos morales impacte en gran medida en estos organismos internacionales, que más que por argumentos morales se encuentran guiados por la lógica de la racionalidad instrumental que únicamente coloca como fin la maximización del beneficio. Será necesario, entonces, un argumento intermedio, un argumento prudencial que bajo los supuestos de la acción estratégica oficie de puente hacia el argumento moral.

Este segundo argumento, de corte prudencial, pretende hacer contar a su favor a ese beneficio que opera como el fin por excelencia. Para presentarlo apelaré a Kant, quien en su caracterización de los imperativos hipotéticos introduce los imperativos hipotéticos asertóricos o de sagacidad, que son los que apuntan a la búsqueda de la felicidad duradera ${ }^{25}$. La búsqueda de la felicidad duradera implica asegurar las condiciones que a mediano y largo plazo permitan continuar optimizando el beneficio que oficia de fin. Esta determinación de la acción es también en términos de medios a fines, pero la diferencia con una racionalidad estratégica cortoplacista está en que el beneficio duradero implicará una consideración del otro que coincidiría con lo exigido por el argumento moral. Es decir, habría una coincidencia entre el argumento moral y el de prudencia, aunque tal coincidencia estaría motivada en forma distinta; en el primer caso la idea de igual dignidad es la causa, mientras que en el segundo lo es la búsqueda del propio beneficio a medio y largo plazo.

Para la construcción de las condiciones posibilitantes de una justicia global, si bien sería deseable que el argumento moral fuera el motivante, es igualmente bienvenido el argumento de prudencia, cuya promoción forma parte de eso que hemos indicado como la parte B de la ética del discurso, y que habilitaría a la acción estratégica de tal forma «[...] que la máxima de su

${ }^{25}$ I. Kant, La fundamentación de la metafisica de las costumbres, op . cit., pp. 66-68. 
acción pudiera considerarse como una norma susceptible de consenso, si no en un discurso real, sí al menos en uno ideal imaginario de todos los afectados de buena voluntad» ${ }^{26}$. A su vez, bajo esta perspectiva, los sujetos se encontrarían comprometidos con la realización a largo plazo y aproximativa de las condiciones de aplicación de la ética discursiva.

Una vez indicado esto, falta entonces señalar por qué el asegurar estas condiciones de justicia global contribuye a esa maximización del beneficio a largo plazo de quienes actúan bajo la lógica de medios a fines.

Las políticas actuales de los organismos internacionales de crédito así como las políticas de subvenciones y las barreras arancelarias son el núcleo duro de las condiciones externas que afectan a los países más pobres, y que determinan en buena medida la imposibilidad de asegurar condiciones de mínimos de dignidad en términos de desarrollo de capacidades básicas para sus ciudadanos. Como ha sido señalado, la causa central para estas políticas se asienta en una lógica de racionalidad instrumental que niega el reconocimiento y cosifica al otro generando un proceso de transvaloración que coloca a aquello que es un fin en sí mismo como un medio y convierte a los medios en fines.

La negación del reconocimiento tiene la consecuencia de provocar el menoscabo de los propios organismos internacionales, puesto que la imposición de medidas económicas o de clausura de posibilidades de comercio que condenan a la pobreza a las sociedades afectadas generan que prácticamente no existan posibilidades de cumplir con los compromisos contraídos. Es una lógica perversa; las medidas pretenden asegurar el mayor beneficio, pero este beneficio, en tanto que es pensado a corto plazo y bajo una lógica cosificadora, socava su propio suelo generando pobreza, exclusión, degradación ambiental y, en definitiva, cancelando la posibilidad de un beneficio continuo.

Por otra parte, las condiciones impuestas por los organismos de crédito y también las generadas por medidas proteccionistas o de subsidios a productos que compiten con los que producen los países más pobres tienen el efecto de exportar a través de la emigración la inestabilidad desde los países pobres al interior mismo de los países más ricos, y este argumento es el que quiero desarrollar con mayor detalle ${ }^{27}$.

En primer lugar debemos atender a que una auténtica preocupación por los problemas de estabilidad de una sociedad debe contar con dos facetas: una que atienda a asegurar la convivencia de distintas concepciones del bien y otra que deberá propiciar la anulación de aquellas situaciones que puedan generar sentimientos de envidia en los sujetos que conviven en la misma

${ }^{26}$ K.-O. Apel, «La ética del discurso como ética de la responsabilidad», op . cit., p. 182.

27 Los daños medioambientales si bien no son exportados a los países ricos como la pobreza y la exclusión a través de la emigración, sí lo son sus consecuencias internacionalizadas en forma de efectos secundarios que se manifiestan a medio plazo. Cf. M. Zürn, «Globale Gefährdungen und internationale Kooperation», Der Bürger im Staat, 45, 1995, pp. 51-54. 
sociedad. Esto último se debe a que, según Rawls, la envidia general, es decir, aquella que es provocada por las desigualdades en la posesión de bienes o recursos, genera el menoscabo del autorrespeto y, en consecuencia, afecta a la condición de sujetos plenamente cooperantes ${ }^{28}$.

Todo aquello que socave el autorrespeto afectará, en primer lugar, a la capacidad de perseguir una concepción del bien y también a la de tener un sentido de justicia; por lo tanto, los sentimientos de envidia estarán destruyendo a los sujetos de la sociedad, en tanto que miembros cooperantes autónomos. Sin autorrespeto nadie tiene un sentido de la propia valía, en el entendido de que la propia concepción del bien sea digna de ser realizada, y de ahí que pase a ser central la eficacia con que los arreglos de justicia aseguren el autorrespeto.

Por lo tanto, las consecuencias de una sociedad inestable a causa de sus arreglos distributivos la afectan internamente porque pierde sujetos cooperantes, pero una consecuencia ulterior es que estas condiciones económicas son una de las fuentes más importantes de la emigración, y esta emigración lleva adosada a los países de destino la inestabilidad. Esta inestabilidad probablemente se generará porque, independientemente de que el país receptor sea rico y justo, la riqueza y las posibilidades distributivas tienen un límite, y un proceso de inmigración masiva tarde o temprano reproducirá las mismas condiciones de marginación y pobreza que han hecho huir al afectado. Ningún país de la Tierra podría soportar una inmigración permanente por lo que en algún momento este fenómeno significará riesgos para la estabilidad social.

De acuerdo con esto, lo que se vuelve necesario es que los organismos de crédito internacional, la OMC y las diferentes formas de cooperación de los países ricos con los pobres se funden en una lógica que opere bajo imperativos prudenciales, asegurando las condiciones que a mediano y largo plazo les permita continuar optimizando el beneficio a unos y asegurando condiciones de bienestar y seguridad a otros.

Pero, como ya se ha dicho, esta lógica prudencial solamente será un puente hacia una lógica moral donde el otro sea reconocido como alguien que tiene valor en sí, de tal forma de asegurar tanto un desarrollo en términos de mínimos de dignidad de las capacidades básicas, como también posibilitar una inclusión universal de los afectados. Y es en esta inclusión radical donde se asienta la ventaja que tiene la lógica moral frente a la lógica prudencial, porque solamente un argumento moral basado en un reconocimiento del otro como alguien que tiene valor en sí permitirá incluir a aquellos que no tienen nada que ofrecer, nada que pueda inducir a que, motivados por el beneficio a medio y largo plazo que se pueda obtener, los organismos implicados cooperen con el desarrollo.

${ }^{28}$ Cf. J. Rawls, A Theory of Justice, Cambridge, Massachussets, Harvard University Press, 1971, pp. 530-534. 
La lógica prudencial solamente involucra a aquellos países que tienen algo que ofrecer, es decir, a aquellos que califican en la solicitud de préstamos, aquellos que tienen recursos y productos que exportar, etc. Pero hay países que no tienen nada de esto, y que, por lo tanto, para una lógica prudencial son inexistentes; no hay ningún beneficio a medio y largo plazo en unas relaciones justas con algunas zonas del planeta que ni siquiera son cosificadas en una relación injusta porque no es atractivo ningún tipo de intercambio con ellos, de ahí que solamente ese valor en sí inherente a las personas sea el que permita potenciar la cooperación y las relaciones justas con ellos. De esta lógica moral y de su promoción dependen estas zonas del planeta que demandan una inclusión radical por la simple razón de que son personas.

En tal sentido, el romper con la lógica cosificadora que hemos presentado oficia como un primer paso en la generación de las condiciones posibilitantes de una justicia global; tanto argumentos prudenciales como morales lo habilitarían. Por su parte, la radical inclusión de todas las regiones del mundo en las relaciones de crédito, comercio y cooperación solamente admite un argumento moral, un argumento basado en la igual dignidad de todos los seres humanos. Ambos procesos son imprescindibles para una justicia global; de ambos depende una toma de conciencia global que instaure una lógica de reconocimiento recíproco que permita bloquear la profecía de Wells. Un mundo de Morlocks y de Eloi, que se temen y se devoran, solamente será evitado a través de una radical inclusión del otro. 